\title{
Synchronization Properties of Two Coupled Multisection Semiconductor Lasers Emitting Chaotic Light
}

\author{
Toni Pérez, Mindaugas Radziunas, Hans-Jürgen Wünsche, Claudio R. Mirasso, and Fritz Henneberger
}

\begin{abstract}
We present numerical simulations describing the dynamics of two multisection semiconductor lasers emitting in a chaotic regime coupled in a master-slave configuration. By changing the current of the passive section of the master laser, we observe a change in the maximum correlation between the outputs of the two systems. These devices are promising candidates for on-off phase-shift keying encryption.
\end{abstract}

Index Terms-Chaos communications, multisection lasers.

C HAOS-BASED communication can be considered as an alternative technique to encode and transmit information at high bit rate in conventional optical communication systems. Recent experiments in an installed optical fiber network have stressed the potential of this technique [1]. Within a chaotic carrier, the information can be encoded in different ways, the most common ones being chaos shift keying [2], amplitude chaos masking [3], and chaos modulation [1]. As an alternative to these traditional schemes, a new promising encryption method termed on-off phase-shift keying (OOPSK) has been proposed recently [4], [5]. It requires two external cavity semiconductor lasers, matched within a certain precision, operating in a chaotic regime unidirectionally coupled in a master-slave configuration. If the external cavities of the lasers are identical, the two outputs synchronize with a high correlation coefficient. However, if the two cavities differ within subwavelength precision, synchronization quality degrades [4]. By periodically, or aperiodically, changing slightly the external cavity of the master laser, the slave becomes synchronized (high correlation coefficient) or unsynchronized (low correlation coefficient) with the master. These two states, synchronized and unsynchronized, allow us to define two bits, " 0 " and " 1 " [5]. The main advantage is that no traces of the message can be guessed either in time or in the frequency domain. Calculations reveal that the

Manuscript received April 19, 2006; revised July 22, 2006. This work is supported by the MEC Acciones Integradas Project HA2005-0051 and MCyT Spain and Feder, by Project TEC-2005- 07799-02-01/MIC and Project FIS200400953, by the EC Project PICASSO IST-2005-34551, and by DAAD Projekt D/05/50619. The work of M. Radziunas was supported by the DFG Research Center Matheon.

T. Pérez and C. R. Mirasso are with the Departament de Física, Universitat de les Illes Balears, E-07122 Palma de Mallorca, Spain (e-mail: claudio@imedea. uib.es).

M. Radziunas is with the Weierstraß-Institut für Angewandte Analysis und Stochastik, 10117 Berlin, Germany.

H.-J. Wünsche and F. Henneberger are with the Institut für Physik, HumboldtUniversität zu Berlin, 12489 Berlin, Germany.

Digital Object Identifier 10.1109/LPT.2006.883179

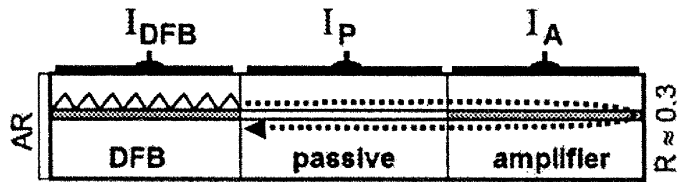

Fig. 1. Schema of a laser with integrated optical feedback. AR: antireflection coating. DFB: $1.55-\mu \mathrm{m}$ DFB laser. Passive: section composed of a material with higher bandgap. Amplifier: integrated $1.55-\mu \mathrm{m}$ amplifier section with an end reflectivity of about $30 \%$.

maximum rate at which information can be encrypted with the OOPSK method depends on the resynchronization time when the system moves from an unsynchronized stated to a synchronized one. This time depends, as well, on the round-trip time in the external cavity [6]. Consequently, external cavity semiconductor lasers would only allow for bit rates $\lesssim 100 \mathrm{M} / \mathrm{s}$ [5]. This limitation could be overcome by using much shorter feedback paths.

In this letter, we consider the limit of ultrashort optical feedback [7] with delay times of only a few picoseconds. Ultrashort optical feedback can be achieved by integrating the feedback path into the semiconductor laser in the framework of a multisection structure [8]. The result is a compact and robust device which, in addition to the short time scales, is an important requirement for possible applications. Multisection lasers are indeed expected to be essential sources for high bit rate OOPSK encryption.

The particular laser structure considered here consists of three sections (see Fig. 1): a single-mode $\lambda=1.55 \mu \mathrm{m}$ distributed feedback (DFB) laser, a passive phase-tuning section of higher bandgap material, and a $1.55-\mu \mathrm{m}$ amplifier section, all with comparable longitudinal dimensions of few $100 \mu \mathrm{m}$. The laser section is antireflection-coated, whereas the reflection from the cleaved facet of the amplifier section provides the desired feedback. Each section is contacted individually and the three dc currents are used to control the dynamics in a specific way. The current $I_{\mathrm{DFB}}$ pumps the laser above threshold; the amplifier current $I_{A}$ allows us to adjust the feedback strength; the injection $I_{P}$ in the passive section influences the refractive index $n_{P}^{\text {eff }}$ and, thus, controls the optical phase shift

$$
\phi_{P}=2 \pi \frac{2 L_{P} n_{P}^{\mathrm{eff}}}{\lambda}
$$

of this section. This functionality yields the required variation of the optical path of the feedback cavity, allowing for several 
wavelengths changes without changing its geometrical length $L_{P}$ (cf. [9, Fig. 4]).

Ultrashort optical feedback conditions lead to two different types of self-pulsations [7], [10], namely undamped relaxation oscillations (ROs) and mode beating (MB) pulsations with frequencies of several gigahertz and several tens of gigahertz, respectively. Such devices can also emit chaotic power with its associated broad spectrum. We shall demonstrate that under these circumstances, synchronization of two chaotic lasers takes place beyond a certain coupling strength. We shall also show a strong dependence of the synchronization quality on the detuning

$$
\Delta \phi_{P}=\phi_{P}^{\text {master }}-\phi_{P}^{\text {slave }}
$$

between the phase shifts of the passive sections of the master and slave lasers, which makes these devices potential excellent sources for OOPSK encryption.

A comprehensive travelling-wave model of such devices, which agrees very well with experimental findings, is carefully described in [10]. ${ }^{1}$ It resolves the spatio-temporal photon-carrier dynamics along the overall cavity of the multisection lasers, including DFB by Bragg gratings and all possible multiple reflections at facets and interfaces. This model is used to search and characterize regimes of chaotic operation. Chaotic emission in a particular point of operation has already been reported experimentally in [10], but no systematics are known until now. Our present investigations show that extended chaotic regimes appear generally at high amplifier currents and low losses in the phase-tuning section. This letter gives representative results for the amplifier current $I_{A}=80 \mathrm{~mA}$ and phase section losses $\alpha_{P}=20 \mathrm{~cm}^{-1}$. In order to clearly distinguish between effects of deterministic chaos and noise, we suppressed spontaneous emission noise in the calculations. Longitudinal spatial hole burning is also neglected to keep the numerical efforts reasonable in case of coupled devices. The quality of chaos emitted by a single device is not influenced by these simplifications. All other parameters are those of the full model used in [10]. Under these conditions, the emission of the device is chaotic for most phase shifts $\phi_{P}$, except in the small interval $0.48<\phi_{P} / 2 \pi<0.62$, where a complex quasi-periodic motion is observed. We will show that the chaotic domain is sufficiently wide for OOPSK. Representative power spectrum and time trace of a single device in chaotic operation are shown in Fig. 2(a) and (b), respectively. Large irregular pulsations can be observed in the optical power while the spectrum is broad and flat except for few peaks on top. The band around $6 \mathrm{GHz}$ corresponds to usual ROs, as already described in [10], where the inversions in distributed feedback (DFB) and amplifier sections oscillate in phase. The low-frequency peak at about $1 \mathrm{GHz}$ is an additional slow antiphase oscillation, which is specific for high-current operation, similar to the known symmetry-breaking oscillations in single-section DFB lasers with longitudinal spatial hole burning [11]. In the present case, this antiphase dynamics causes power dropouts with subsequent extremely high spikes, which interrupt the few-gigahertz RO pulsations. The $40-\mathrm{GHz}$ band belongs to

\footnotetext{
${ }^{1}$ Information about the software used can be found at http://www.wiasberlin.de/software/ldsl.
}
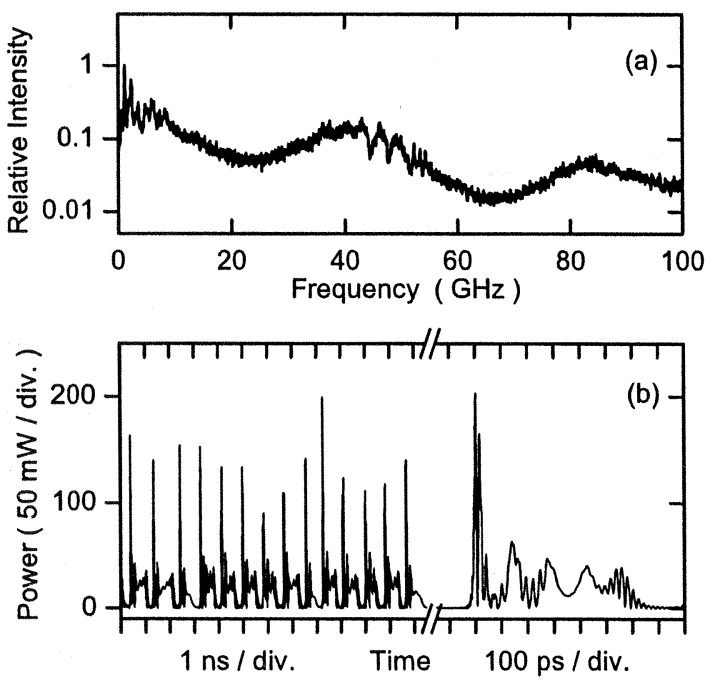

Fig. 2. Chaotic emission of a single multisection laser. (a) Power spectrum. (b) Representative time trace. The phase shift of the passive section is $\phi_{P}=$ $1.94 \pi \bmod 2 \pi$

broadened mode-beating pulsations [10]. All the incommensurable vibrations interfere with each other and give rise to the wide chaotic band with frequencies covering several orders of magnitude.

An important characteristic of the optical output is the autocorrelation time $\tau_{c}$. We calculate it from the autocorrelation function $\Gamma_{i i}(\tau)$ of the either the master $(i=m)$ or the slave $(i=s)$ laser as

$$
\tau_{c}=\int_{0}^{\infty} d \tau \Gamma_{i i}^{2}(\tau)
$$

The correlation function between two output power series $P_{i}(t)$ and $P_{j}(t)$ is defined as

$$
\Gamma_{i j}(\tau)=\frac{\left\langle\left(P_{i}(t)-\left\langle P_{i}\right\rangle\right)\left(P_{j}(t+\tau)-\left\langle P_{j}\right\rangle\right)\right\rangle}{\sqrt{\left\langle\left(P_{i}(t)-\left\langle P_{i}\right\rangle\right)^{2}\right\rangle\left\langle\left(P_{j}(t)-\left\langle P_{j}\right\rangle\right)^{2}\right\rangle}} .
$$

The autocorrelation time calculated on base of these definitions is as short as few tens of picoseconds. This very short time is an important prerequisite for the attempted high-speed operation. It is worth mentioning that this value is representative for the whole domain of phase shift $\phi_{P}$ with chaotic emission.

Once a well-developed chaotic regime is identified, two identical devices are coupled in a master-slave configuration. To quantify the degree of synchronization, we calculate the maximum of the cross-correlation function $\Gamma_{m s}$ between master and slave systems from emitted powers filtered with a Butterworth 25-GHz low-pass filter of order 5. The filtering suppresses the high-frequency MB oscillations which do not synchronize well. In possible future experiments, similar filtering is introduced anyway by the limited bandwidth of optoelectronic conversion. Synchronization is expected to depend on the coupling strength $\kappa_{c}$, which is the percentage of the master power coupled into the slave. For low values of $\kappa_{c}$, we anticipate uncorrelated chaotic output. The correlation should improve with increasing coupling and should reach high values for large $\kappa_{c}$. The plot in Fig. 3 confirms this scenario for the considered case of identical devices. At small $\kappa_{c}$, the synchronization quality is poor but it 


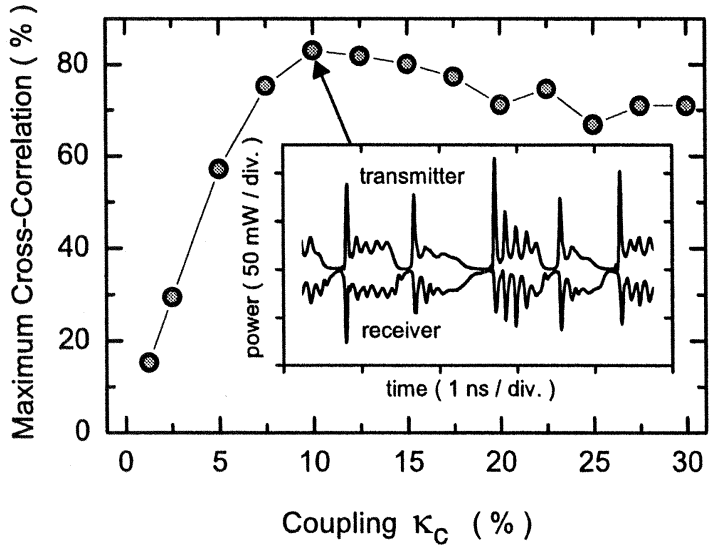

Fig. 3. Dependence of the maximum of the cross-correlation function $\Gamma_{m s}(\tau)$ on the coupling strength $\kappa_{c}$. The passive phase shifts $\phi_{P}$ of transmitter and receiver are $1.84 \pi$ and $1.44 \pi$, respectively. Inset: Low-pass filtered transients of the synchronized transmitter and receiver for $\kappa_{c}=10 \%$. The receiver amplitude is inverted for better comparison.

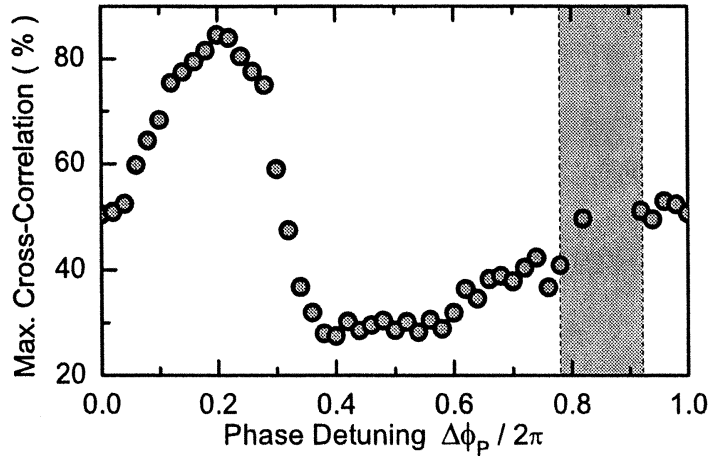

Fig. 4. Maximum of the cross-correlation function $\Gamma_{m s}(\tau)$ versus the detuning $\Delta \phi_{P}$ between the passive phase shifts of transmitter and receiver laser. The coupling strength is $\kappa_{c}=10 \%$. Bullets: calculated from 34-ns-long time traces. Gray area between dashed lines: quasi-periodic emission of the transmitter.

improves rapidly with increasing $\kappa_{c}$. Beyond $\kappa_{c} \approx 30 \%$, the degree of synchronization saturates at about $80 \%$. It is worth mentioning that synchronization does not occur at the flying time between master and slave lasers but it is slightly shifted (about $10 \mathrm{ps)}$ in agreement with recent results [12].

To check the potentiality of this configuration for the OOPSK encryption, we have to analyze the dependence of the synchronization degree on the detuning $\Delta \phi_{P}$ between the phase shifts in master and slave lasers [cf. (2)]. The plot of the maximum of the cross-correlation function $\Gamma_{m s}(\tau)$ versus $\Delta \phi_{P}$ in Fig. 4 exhibits a variation between about $30 \%$ to $80 \%$. This range is already a sufficiently high contrast for OOPSK and compares to the one presented in [5], although a more detailed bit-error-rate analysis is required. Its applicability, moreover, will also depend on the resynchronization time which we have roughly estimated to be below 1-2 ns.

We expect that optimized structures will improve these operation characteristics. In Fig. 4, it can also be noticed that the maximum correlation does not appear at a zero detuning but at $\Delta \phi_{P} \approx 0.4 \pi$. Although not understood yet in detail, this effect is not surprising. The power injected into the receiver not only triggers the synchronization but also changes the average conditions of operation. It acts like a detuning of pump currents, which is partly compensated by the phase detuning.

In conclusion, we have numerically demonstrated that two multisection lasers with internal amplified optical feedback, appropriately coupled in a master-slave configuration, are excellent potential sources for OOPSK encryption. When the passive phase shift $\phi_{P}$ in the master laser gets detuned from that of the slave laser, we observe a periodic variation of the synchronization with a $2 \pi$ periodicity. Under appropriate coupling strengths between master and slave systems, we obtain a maximum correlation coefficient of about $80 \%$ and a minimum of about $30 \%$, values well suited for an adequate OOPSK modulation. These encouraging results have been obtained with parameter-identical master and slave. We are optimistic that the flexibility of the device will allow us to compensate for small parameter mismatches, although we expect that large mismatches cannot be compensated and very similar devices, as those required when using external cavity lasers, are still essential.

\section{REFERENCES}

[1] A. Argyris, D. Syvridis, L. Larger, V. Annovazzi-Lodi, P. Colet, I. Fischer, J. Garcia-Ojalvo, C. R. Mirasso, L. Pesquera, and K. A. Shore, "Chaos-based communications at high bit rates using commercial fiberoptic links," Nature, vol. 438, pp. 343-446, 2005.

[2] C. Mirasso, J. Mulet, and C. Masoller, "Chaos shift keying encryption in chaotic external-cavity semiconductor lasers using a single-receiver scheme," IEEE Photon. Technol. Lett., vol. 14, no. 4, pp. 456-458, Apr. 2002.

[3] M. J. Ogorzalek, "Timing chaos-Part I: Synchronization," IEEE Trans. Circuits Syst. I, Reg. Papers, vol. 40, no. 10, pp. 693-699, Oct. 1993.

[4] M. Peil, T. Heil, I. Fischer, and W. Elsäßer, "Synchronization of chaotic semiconductor laser systems: A vectorial coupling-dependent scenario," Phys. Rev. Lett., vol. 88, p. 174101, 2002.

[5] T. Heil, J. Mulet, I. Fischer, C. R. Mirasso, M. Peil, P. Colet, and W. Elsäßer, "ON/OFF phase shift keying for chaos-encrypted communication using external-cavity semiconductor lasers," IEEE J. Quantum Electron., vol. 38, no. 9, pp. 1162-1170, Sep. 2002.

[6] R. Vicente, T. Pérez, and C. Mirasso, "Open- versus closed-loop performance of synchronized chaotic external-cavity semiconductor lasers," IEEE J. Quantum Electron., vol. 38, no. 9, pp. 1197-1204, Sep. 2002.

[7] O. Ushakov, S. Bauer, O. Brox, H.-J. Wünsche, and F. Henneberger, "Self-organization in semiconductor lasers with ultra-short optical feedback," Phys. Rev. Lett., vol. 92, no. 043902, 2004.

[8] S. Bauer, O. Brox, J. Kreissl, G. Sahin, and B. Sartorius, "Optical microwave source," Electron. Lett., vol. 38, no. 7, pp. 334-335, 2002.

[9] U. Bandelow, H. J. Wünsche, B. Sartorius, and M. Möhrle, "Dispersive self- $Q$-switching in DFB lasers-Theory versus experiment," IEEE J. Sel. Topics Quantum Electron., vol. 3, no. 2, pp. 270-278, Apr. 1997.

[10] S. Bauer, O. Brox, J. Kreissl, B. Sartorius, M. Radziunas, J. Sieber, H.-J. Wünsche, and F. Henneberger, "Nonlinear dynamics of semiconductor lasers with active optical feedback," Phys. Rev. E, vol. 69, p. 016206, 2004.

[11] A. J. Lowery, "Dynamics of SHB-induced mode instabilities in uniform DFB semiconductor lasers," Electron. Lett., vol. 29, pp. 1852-1854, 1993.

[12] S. Peters-Flynn, P. S. Spencer, S. Sivaprakasam, I. Pierce, and K. A. Shore, "Identification of the optimum time-delay for chaos synchronization regimes of semiconductor lasers," IEEE J. Quantum Electron., vol. 42, no. 4, pp. 427-434, Apr. 2006. 